\title{
Agent-based coalition formation in disaster response applications
}

\author{
Ladislau Bölöni, Majid Ali Khan and Damla Turgut \\ School of Electrical Engineering and Computer Science \\ University of Central Florida \\ Orlando, FL 32816, \\ Email: 1boloni@eecs.ucf.edu,majid@ hector.cs.ucf.edu, turgut@eecs.ucf.edu
}

\begin{abstract}
We present an agent-based coalition formation approach for disaster response applications. We assume that agents are operating in a dynamic and dangerous environment, and they need to form convoys to efficiently traverse unsafe areas and randez-vous at task locations. We assume a dynamic model of multilevel coalition formation where agents can dynamically join and leave the convoy, and the goals of the convoy (as reflected in its physical path and schedule) is determined by its current members. We demonstrate our approach in a simulation study located in the environment of New Orleans in the hurricane Katrina aftermath.
\end{abstract}

\section{Introduction}

Efficient disaster response requires participants to form teams and coordinate their actions. This process is complicated by a variety of factors:

Dynamic, unpredictable and dangerous environment. In the immediate aftermath of a disaster (such as the hurricane Katrina in New Orleans or the Asian Tsunami) previously safe areas might turn into unsafe or unaccessible. The environment might contain new sources of danger in the form of natural obstacles (damaged buildings) or even hostile agents (such as looters or stray dogs).

Dynamic tasks. In rescue missions, tasks appear unpredictably. The discovery of a wounded person at a dangerous location creates a new task with specific logistics, protection and medical facets. In severe disasters, the number of tasks can greatly exceed the available resources.

Dynamic teams and collaboration patterns. Although some of the disaster management teams are pre-established, trained together and have a clear pattern of command and control, many teams are assembled on an ad hoc basis, as a response to emerging tasks. Teams are composed from heterogeneous groups of entities: persons, vehicles, service animals, and so on. Team members might not report to the same chain of command, might have communication problems and their interests might not be completely aligned. For instance, the state police and guerilla groups might cooperate in a rescue operation but resume hostilities after the emergency.

Our research group at the Networking and Mobile Computing (NetMoc) laboratory at University of Central Florida is working on a negotiation based coalition formation approach which can be used to assemble ad hoc coalitions in an emergency management scenario. In this paper, we are concentrating on the negotiation regarding convoy formation for mobility in a dangerous environment. The convoy formation approach, which is based on physical destinations needs to be complemented with a decision process based on assigning tasks based on the roles the agents are able to assume in a team.

\section{World model}

The environment considered in this paper assumes a 2dimensional geographic area, where we identify: safe areas which are traversable by any vehicle and convoy, danger areas which are traversable only by convoys and inaccessible areas. The model can be extended in a straightforward way to involve more than three area types which affect the movement of the vehicles in a variety of ways (such as slowing down, requiring higher energy consumption, and so on). In this environment, we consider the actions of a set of $\mathrm{em}$ bodied agents, which have a well-defined physical location and movement capabilities. In practice, these agents can be "RAP" (Robots, Agents and/or Persons). The goal of every agent is to execute a certain task at a destination location.

The time to reach the destination can be improved by the formation of convoys. In certain cases, the agent can not reach the destination except through joining convoys. We assume the agents self-interested but honest; the agents keep their negotiated commitments. The embodied agents are using message based communication, which can be either point to point or broadcasted to all other agents in the 
transmission range.

Negotiation is the process by which a group of agents come to a mutually acceptable agreement on some matter [6]. In our scenario, the subject of negotiation is the joining and leaving convoys, and the adaption of the path of the convoy to the requirements of the agent. The agents are exchanging a set of offers, based on their offer construction strategies. The other party is using its offer evaluation strategy to make a decision, which can be either to accept the offer, send a counteroffer or terminate the negotiation. Two subprotocols (for joining and leaving convoys) describe the message flows for the different negotiation processes.

While this model is common for any kind of negotiation processes, the fact that the negotiation happens in real-time in the physical world, creates a set of new requirements. The negotiation needs to be time constrained, i.e. the time allotted to the concrete flow of messages needs to be limited. The agents being in constant movement, they will be in their communication range for a limited amount of time. The negotiation needs to be fail safe due to the frequent loss of messages either due to temporary causes, or because a vehicle got out of the communication range during the negotiations. It is especially problematic if a negotiation is interrupted with the parties having a different view of the outcome of the negotiation. The negotiation has to be deadline oriented because the offers made during negotiations can become obsolete. For instance, if an agent A makes an offer to join convoy $\mathrm{B}$ at location $\mathrm{X}$ and time $\mathrm{t}$, there is a limited temporal window of opportunity when this rendez-vous can take place. Thus every offer needs to carry a timestamp and an expiration date. Also, since one vehicle can take part in several negotiations simultaneously, the negotiation process should make sure that it does not commit to contractual binding with more than one vehicles at the same time.

We define a convoy as a coalition of embodied agents which agreed on a common path and schedule. Normally, the agents of the coalition have a common location and speed; however, from a logical perspective, we consider an agent which has agreed to join a convoy and it is on its way to a rendez-vous point as part of the convoy.

Convoys have a hierarchical structure, and may contain sub-convoys. For the sake of uniformity, we will consider that individual vehicles are being part of single-vehicle convoys. Formally, a convoy $C$ is described by a set of convoys $S=\left\{C_{1}, \ldots C_{n}\right\}$, a leader agent $A_{L} \in S$, and a set of commitments $G=\left\{g_{1}, \ldots g_{n}\right\}$. The set of commitments are usually expressed as constraints on the path of the convoy. The role of the leader is to negotiate on behalf of the convoy and to determine its path, taking into account its previous agreements $G$. Although the embedded convoys maintain their leader and set of agreements, the path of the convoy is determined exclusively by the leader of the outermost convoy. The negotiation protocols need to ensure that the agreement sets of the subconvoys are compatible with the agreements of the embedding convoy.

The commitment of the convoys are related to visiting locations and can be classified as "before" (B) and "after" (A) commitments. A "before commitment" $B(L, t)$ commits the convoy to arrive to location $L$ not later than time $t$. An "after commitment" $A(L, t)$ commits the convoy to leave location location L not sooner than time $t$ (if the convoy reaches that location sooner, it can, of course wait at the location).

We will call a commitment $C_{1}$ stronger than a commitment $C_{2}$ and denote it $C_{2} \subset C_{1}$ if every set of actions which satisfies $C_{1}$ also satisfies $C_{2}$.

\section{Theorem 1}

$$
\begin{aligned}
& \forall L, t_{1}<t_{2} \Rightarrow B\left(L, t_{2}\right) \subset B\left(L, t_{1}\right) \\
& \forall L, t_{1}<t_{2} \Rightarrow A\left(L, t_{1}\right) \subset A\left(L, t_{2}\right)
\end{aligned}
$$

We leave the proof of this theorem as an exercise to the reader.

\section{Convoy formation mechanism}

The convoy joining mechanism is inherently asymmetric, even if it takes place between two single-agent convoys. The leader agent of the first convoy will become the leader of the resulting convoys. There is an asymmetry in the negotiation interests of the main convoy and the joining agent or convoy.

Let us now consider the lifecycle of the embodied agents, and the objectives of the negotiation. The simplified state diagram of the lifecycle of the agent is shown in Figure 1. The default state of the agent is to move independently towards its destination $(x, y)$. Whenever an agent detects the presence of a convoy in its vicinity, it starts a negotiation process. If the negotiation is successful, the agent moves to join the convoy at a rendez-vous point. From then on, the agent moves with the convoy, until the pre-agreed leave point is reached. At the leave point, the agent leaves the convoy, and moves independently to its destination. Even while the agent is in the convoy, it might start negotiations with other convoys, or simply consider to leave the convoy on its own. If the agent wants to leave the convoy before the leave point, the agent needs to negotiate this with the convoy leader. If the negotiation is successful, the agent leaves the convoy, and it is free to follow its separate path to the destination, or join a different convoy.

Let us consider the objective of the negotiation. We will denote with $\tau_{C}\left(L_{1}, L_{2}\right)$ the time it takes for convoy $\mathrm{C}$ to move from location $L_{1}$ to location $L_{2}$. In the simplest case, at time $t$ an agent $A$ with the destination $D_{A}$ and current location $L_{A}$ considers joining a convoy $C$, which has a current set of commitments $G$. The agent has its current expected arrival time $t_{A}=t+\tau_{A}\left(L_{A}, D_{A}\right)$. In the first approximation, the agent would join the convoy if it can add 


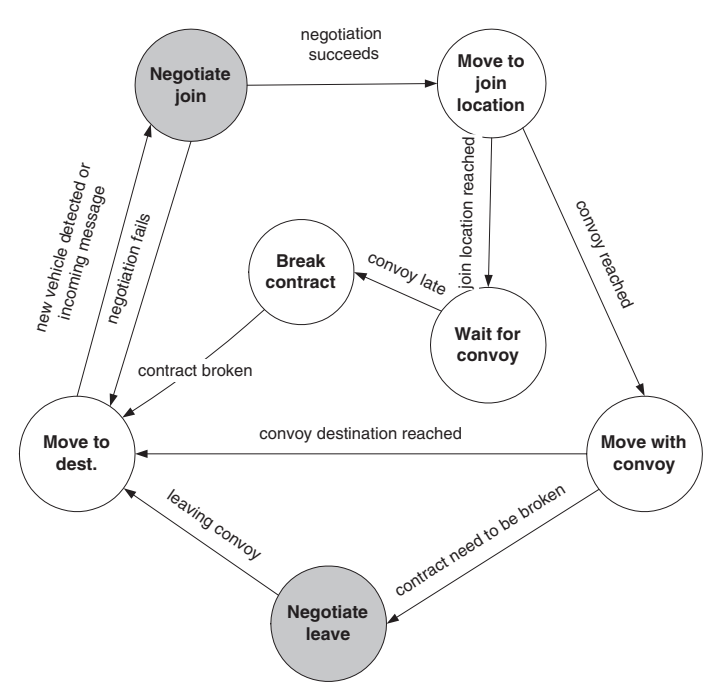

Figure 1. The simplified lifecycle of an embodied agent which moves towards the destination optionally joining convoys.

to its list of commitments an agreement $B\left(D_{A}, t_{A}^{\prime}\right)$ with $t_{A}^{\prime}<t_{A}$, that is, it can reach its final destination faster. However, even if this agreement is not feasible, it might be worth for the agent to join the convoy up to an intermediate location $P$, called the leave point. A sufficient condition for the agent to be worth joining the convoy until leave point $L_{\text {leave }}$ is to have a commitment $B\left(L_{\text {leave }}, t_{\text {leave }}\right)$ such that $t_{\text {leave }}+\tau_{A}\left(L_{\text {leave }}, L_{A}\right)<t_{A}$. This is however not a necessary condition; the agent might plan ahead for joining a different convoy after leaving the current convoy at $P$.

A successful negotiation for an agent joining a convoy will add two commitments to the convoys set of commitments: a commitment $A\left(L_{\text {join }}, t_{j o i n}\right)$ for the join location of the agent, and a commitment $B\left(L_{\text {leave }}, t_{\text {leave }}\right)$ for the leave location of the agent.

Thus, the negotiation between the convoy and the agent is a multi-objective negotiation, with the $\left\langle L_{\text {join }}, L_{\text {leave }}, t_{\text {join }}, t_{\text {leave }}\right\rangle$ quadruplet being the minimal negotiation set. In addition to these four objects, the negotiation might involve cost, penalties for contract breaking or other temporal constraints. In the following, we will briefly discuss the four negotiation objectives and their interrelationships.

$L_{\text {join }}$ the location to join the convoy. The interest of the agent is to negotiate a join location which is as close to its current location as possible, or to be in the general direction of the destination. The choices of the convoy are (in order of preference) (a) to negotiate a join location $L$ for which he already has an $A(J, t)$ commitment, (b) a location for which it has a $B(J, t)$ commitment, (c) a location which is on the current projected path of the convoy and (d) a location which is close to the current projected path of the convoy. Intuitively, (a) does not involve any new commitment for the convoy (if it manages to negotiate a join time earlier or the same as the previous commitment), (b) requires only a temporal commitment, without new restrictions on the path of the convoy. A location of type (c) restricts the ability of the convoy to change its path (although its current path remains valid), while a point of type (d) requires the convoy to change its path. These preferences will be inevitably reflected in the negotiation strategy of the convoy. One additional complexity is the number of negotiation choices. While the points of type (a) and (b) are coming from a limited set of discrete choices, the points (c) are coming from a one-dimensional while points (d) from a two dimensional continuum, limited only by the resolution of the raster maps on which the systems operate. This leads to an unrealistically large negotiation space. To reduce the negotiation space to a more realistic size, we chose to identify segment locations on the convoy path. By restricting the choice of the rendez-vous and leave locations to the segment points, we guarantee that the negotiation happens over a discrete set of choices. These locations have special properties, such as they are situated on the intersection between safe and danger zones, or on the intersection between the convoys path and the agents path. In the first pass, we can eliminate the points which are not feasible because of one of the following reasons:

(i) the convoy has already passed that segment location

(ii) the vehicle cannot reach the segment location before the convoy passes it

(iii) the segment location is unreachable by the vehicle (e.g. if it lies within a safe region surrounded by unreachable and danger zones)

$t_{\text {join }}$ - the joining time. Once the agent and the convoy had identified its join location they need to negotiate the join time. In broad lines, the agent negotiates for the latest possible join time (to increase its safety margin in getting there), while the convoy for the earliest time (because that minimizes its commitment in waiting for the agent). The join time has to be at least the minimum time needed by the agent to reach the join point (the convoys minimal arrival time is not strictly relevant, as its commitment is to leave after the negotiated time). This is a simple linear negotiation, which (for all other negotiation objectives fixed) can be resolved with a monotonic concession protocol with Zeuthen strategy [11]. However, we need to observe that once the hard requirement of $\tau\left(L_{\text {current }}, L_{\text {join }}\right)<t_{\text {join }}-t_{\text {current }}$ is met, the rest of the negotiation is only about safety margins. Thus, an agent is more likely to concede in this parameter, which does not affect its predicted performance.

$L_{\text {leave }}$ - the leave location. For this location, the interest of the agent is to negotiate a location as close as possible 
to its final destination (except the case when it is planning to join another convoy on the leave location). The interest of the convoy are, in the order of preference (a) a location for which an existing B commitment exists, (b) a location for which an existing A commitment exists, (c) a point on the current planned path and (d) a point close to the current planned path. Note that the order of preferences for types (a) and (b) is reversed for this point compared to the join point. Similarly to the join location, for types (c) and (d) we consider segmentation approaches. An additional problem which needs to be considered by the convoy is that at every leave location the resources of the convoy are diminished and at the last leave location we end up with two independent agents, not with a convoy and an agent. Thus, the interest of the convoy might be to negotiate for leave points as far down as possible on its projected path. The ideal organization is a single leave point where all the participant agents leave for their individual destinations.

$t_{\text {leave }}$ - the leave time. This parameter represents the guaranteed arrival time at the leave location. $t_{\text {leave }}$ has a lower bound, limited by the physical time a convoy needs to reach the location on the optimal path, while still meeting its other commitments. The upper bound of this parameter is given by the limit at which it is not worth anymore for an agent to join the convoy $t_{\text {leave }}^{\text {upper }}=t_{\text {current }}+\tau_{\text {agent }}\left(L_{\text {current }}, L_{\text {destination }}\right)-$ $\tau_{\text {agent }}\left(L_{\text {leave }}, L_{\text {destination }}\right)$. Evidently, the interest of the agent is an earliest possible time - preferably the lower bound. The interest of the convoy is to minimize its commitment, by committing to as late time as possible. By accepting the lower bound, the convoy is essentially committing that it will not change its current path. This limits its ability to accommodate agents joining in the future.

\section{Experimental results}

To test our coalition formation algorithms, we have tested them through simulating a realistic scenario based on the environment of the hurricane Katrina flooded New Orleans. The agents were implemented in the YAES simulation environment [5]. The physical environment is a $0.9 \times 1.5$ $\mathrm{km}$ large area of New Orleans, represented through a satellite photo with a resolution of 2 meters/pixel, obtained from Google Maps (Figure 2). The safe, unsafe and unaccessible areas were obtained partially from image processing, and partially manually edited. The scenario considers the movement of three agents from their starting points Start-1, Start-2 and Start-3 to their destination points Dest-1, Dest2 and Dest- 3 respectively. We assume that the agents are moving at the very slow speed of $1.2 \mathrm{~km} / \mathrm{h}$. The latency in preparing and delivering the messages is assumed to be 1.2 seconds, while the communication range of the agents is 100 meters - realistic for walkie-talkie type device in an urban environment.
The negotiation happens in real time and the agents keep moving while negotiating. Thus, offers can become invalid if they are answered too late, as both agents have changed their positions. To limit the time taken by the negotiation process, we introduce the negotiation limit $\epsilon$. This limit is applied only to the part of the negotiation dealing with the temporal values $t_{\text {join }}$ and $t_{\text {leave }}$; the agents have an unlimited time to negotiate join and leave locations. For this simulation, we have chosen a value of $\epsilon=30$ seconds, which limits the agents to at most 25 exchanged offers (since 25 offers* 1.2 s/offer $=30$ seconds).

Let us now evaluate the flow of the negotiation. As agents start moving towards their destination, their initial path goes through the path identified by note 1 on Figure 2 . After traveling some distance, Agent-1 and Agent-2 come within communication range of each other and start negotiations for coordinating their movements. Table 1 shows the offers and counter offers made during this negotiation process. Please note that the location of the agent is shown as distance in meters from the top left corner of the map.

Offers 1 and 3 are rejected by Agent- 1 because it cannot satisfy the leave constraint; the time it takes to reach the proposed join location and then move with Agent-2 as convoy does not provide any improvement with respect to Agent1's original path. Similarly Agent-2 rejects the first offer from Agent-1. However, the fourth offer, which originated from Agent- 1 for Agent- 2 is accepted by Agent- 2 because it satisfies both join location and leave location constraints. As stated earlier, after agreeing on join and leave locations, the negotiating parties have $\epsilon$ time to negotiate over join and leave time. The actual time for Agent- 1 to reach join location $(132,472)$ is 1.58 minutes. The time conveyed through negotiation object is $1.58+0.5=2.08$ minutes. The negotiation space for join time for Agent-1 is [2.08, 2.58]. Similarly the time to reach leave location is $48.42+0.5=$ 48.92 minutes. The negotiation space for leave time for Agent- 1 is $[48.42,48.92]$. The time it will take the Agent2 to reach join location is 2.38 minutes. The negotiation space for Agent-2 join time is therefore [2.38, 2.88] and for the leave time is $[48.44,48.94]$. During time negotiations, Agent-2 gradually increases its offer of join time and decreases leave time. Agent-1 does the opposite thing and after some exchanges, the parties agree on the constraints (A((132, 472), 2.38$), B((1390,390), 48.84)$. Please note that the negotiation ending criteria in this case was reaching the upper limit of Agent-1 on join time. In this particular instance, Agent- 2 was able to increase join time by 0.5 minutes, but the leave time was not decreased.

So Agent-1 and Agent-2 meet at join location $(132,472)$ after around 2.38 minutes of reaching agreement and form a convoy. They also agree that Agent- 2 will leave the convoy at location $(1390,390)$ after around 48.8 minutes. Note that the time for Agent-2 to reach its location using only the 


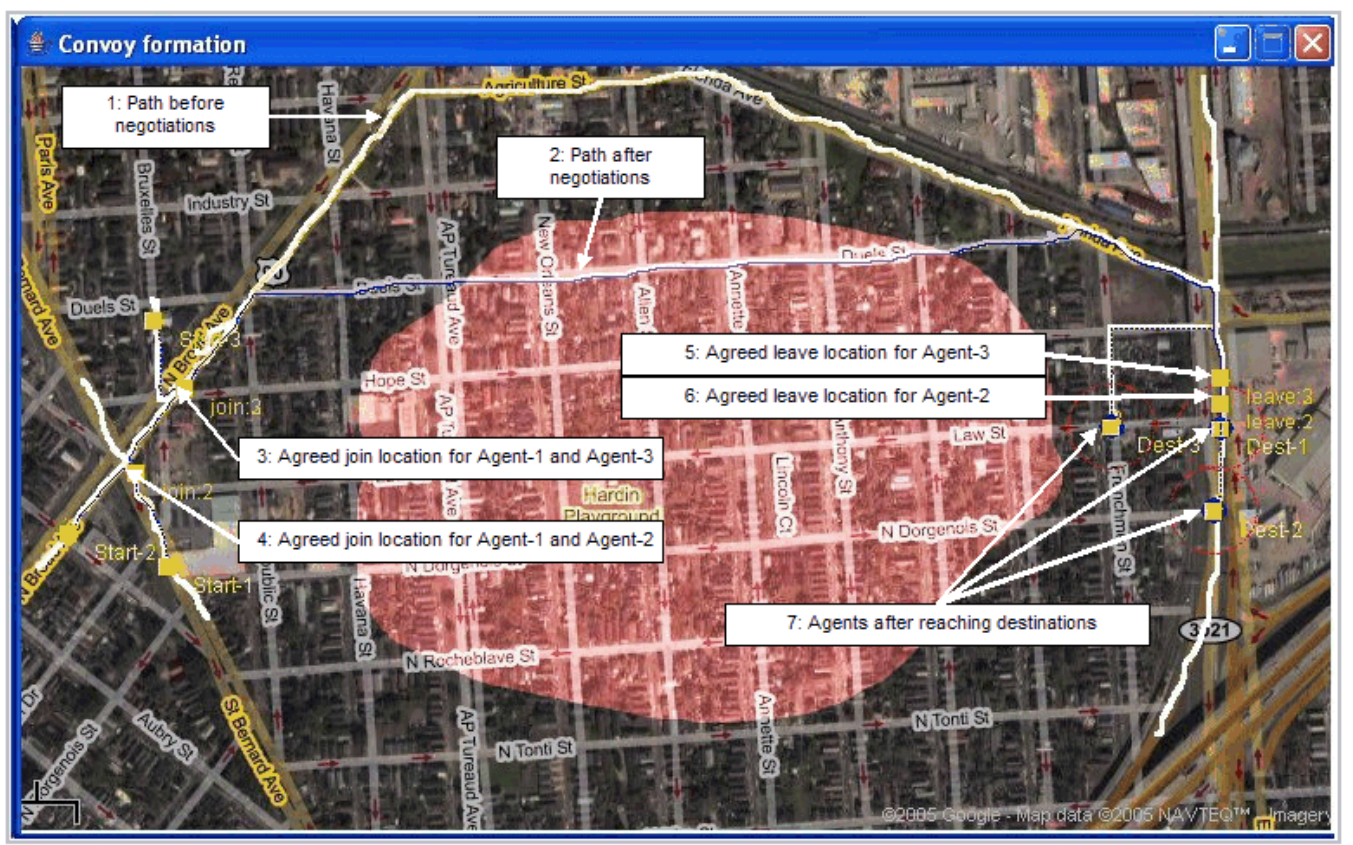

Figure 2. An example run of the coalition formation algorithm, on a map representing an area of New
Orleans flooded in the aftermath of Hurricane Katrina. The area marked in the center of the map is a
danger area, which can not be traversed by individual agents, but is accessible for convoys.

safe zones was 55.7 minutes. The time to reach the same location using the convoy is 52.3 minutes (which includes join time, convoy time and time to reach its destination from leave location).

The convoy then encounters Agent- 3 during travel and a similar negotiation process takes place between convoy leader (i.e. Agent-1) and Agent-3. This process results in another agreement being formed between convoy and Agent-3. The join and leave locations of this agreement are also identified on Figure 2.

The convoy, now consisting of three agents, moves through the danger region and the agents leave the convoy at the agreed leave locations.

It can be seen from Figure 2 that the leave location of the agents is not an optimal location. The reason for that are two fold. First of all, the agents do not know about each other's path. So they cannot determine optimal leave location on other agent's path. Secondly, an approximation could be obtained by providing feedback to the offering party to successively decrementing the leave location. But this would require increased negotiation steps and computational time. Also, by the time we agree on the leave constraint, the join constraint would become invalid.

\section{Related Work}

The field of multi-agent negotiation is influenced by economic models, game theory and artificial intelligence. Jennings et. al [6] defines negotiation as a search process where multiple agents search through the negotiation space to reach agreements and discusses several negotiation models including game theoretic, heuristic based and argumentation based models. Kraus [7] provides a more in depth study of strategic negotiations in multi-agent environments.

Coalition formation between agent residing in the physical world has been the object of study of collaborative robotics. One recent effort is the DARPA Software for Distributed Robotics (SDR) program where researchers from SRI International, Stanford University, the University of Washington, and ActivMedia Robotics are designing and implementing a computational framework for the coordination of large robot teams, consisting of at least 100 small, resource limited mobile robots (CentiBOTS) on an indoor reconnaissance task. The Robocup robotic soccer challenge is also a source of research in coalition formation schemes [4]. Alami et al [1] presents a scheme of operating a large number of mobile robots using plan merging paradigm. Their scheme is based on local knowledge and incremental planning in a distributed manner. They attempt to resolve the spatial movement conflicts between mobile 


\begin{tabular}{|l|l|l|l|l|l|}
\hline Offer No. & Sender & Receiver & $\begin{array}{l}\text { Location of } \\
\text { the sender }(m)\end{array}$ & $\begin{array}{l}\text { Join constraint } \\
\langle(m, m), \text { min }>\end{array}$ & $\begin{array}{l}\text { Leave constraint } \\
\langle(m, m), \text { min }>\end{array}$ \\
\hline 1 & Agent-2 & Agent-1 & $(86.6,501.2)$ & $A((122,462), 1.93)$ & $B((1390,496), 54.26)$ \\
\hline 2 & Agent-1 & Agent-2 & $(159,531.2)$ & $A((134,492), 1.784)$ & $B((1390,400), 49.13)$ \\
\hline 3 & Agent-2 & Agent-1 & $(87,500.6)$ & $A((132,442), 2.46)$ & $B((1390,486), 53.84)$ \\
\hline 4 & Agent-1 & Agent-2 & $(158.8,530.6)$ & $A((132,472), 1.98)$ & $B((1390,390), 48.842)$ \\
\hline 5 & Agent-2 & Agent-1 & $(87.4,499.9)$ & $A((132,472), 2.18)$ & $B((1390,390), 48.742)$ \\
\hline 6 & Agent-1 & Agent-2 & $(158.7,529.8)$ & $A((132,472), 2.08)$ & $B((1390,390), 48.842)$ \\
\hline 7 & Agent-2 & Agent-1 & $(87.7,499.2)$ & $A((132,472), 2.28)$ & $B((1390,390), 48.742)$ \\
\hline 8 & Agent-1 & Agent-2 & $(156.2,529.1)$ & $A((132,472), 2.18)$ & $B((1390,390), 48.842)$ \\
\hline 9 & Agent-2 & Agent-1 & $(88.8,498.1)$ & $A((132,472), 2.38)$ & $B((1390,390), 48.742)$ \\
\hline $10 *$ & Agent-1 & Agent-2 & $(157.1,528.6)$ & $A((132,472), 2.38)$ & $B((1390,390), 48.842)$ \\
\hline
\end{tabular}

Table 1. The offers exchanged between Agent-1 and Agent-2

robots. Although we have a similar problem domain, our effort differs in that 1) we use negotiations for coordinating the movement and 2) our general goal has been to make mobile agents to agree on a meeting and leaving location rather than resolving the spatial movement conflicts.

Although team formation is frequently considered a centralized activity, where a manager assembles teams based on optimization criteria, several research efforts have dealt with negotiation based team formation models. The DARPA Autonomous Negotiating Teams (ANTS) program was one of the focus points of this effort. Some of these papers are concerned with a multi sensor target tracking problem $[8,10]$. Sariel and Balch [9] use an auction based approach for task allocation in multiple robot map exploration problem.

The CoAX - Coalition Agents Experiment series demonstrated the utility of agent technology for coalition operations in a series of technology integration experiments $[2,3]$.

\section{Conclusions}

This paper presented an agent-based coalition formation approach for disaster response applications. We find that many of the formal negotiation models are applicable, but the constraints of the physical world, such as temporal and spatial distances, communication constraints, as well as real-time operation requirements add new requirements. Future work include extending the coalition model to task oriented domains, development of improved algorithms for real-time operation as well as implementation on physical embodied agents.

\section{References}

[1] R. Alami, S. Fleury, M. Herrb, F. Ingrand, and S. Qutub. Operating a large fleet of mobile robots using the plan-merging paradigm. In IEEE International Conference on Robotics and Automation (ICRA'97), pages 2312-2317, 1997.

[2] D. Allsopp, P. Beautement, J. Bradshaw, E. Durfee, M. Kirton, C. Knoblock, N. Suri, A. Tate, and C. Thompson. Coalition agents experiment: Multi-agent co-operation in an international coalition setting. Special Issue on Knowledge
Systems for Coalition Operations (KSCO), IEEE Intelligent Systems, 17(3):26-35, May/Jun 2002.

[3] D. Allsopp, P. Beautement, M. Kirton, A. Tate, J. Bradshaw, N. Suri, and M. Burstein. The coalition agents experiment: Network-enabled coalition operations. Special Issue on Network-enabled Capabilities, Journal of Defence Science, 8(3):130-141, Sep 2003.

[4] J. Anderson, B. Tanner, and J. Baltes. Dynamic coalition formation in robotic soccer. In Proceedings of the AAAI04 Workshop on Forming and Maintaining Coalitions and Teams in Adaptive Multiagent Systems, San Jose, CA, July 2004.

[5] L. Bölöni and D. Turgut. YAES - a modular simulator for mobile networks. In Proceedings of the 8-th ACM/IEEE International Symposium on Modeling, Analysis and Simulation of Wireless and Mobile Systems MSWIM 2005, October 2005.

[6] N. R. Jennings, P. Faratin, A. R. Lomuscio, S. Parsons, C. Sierra, and M. Wooldridge. Automated negotiation: prospects, methods and challenges. International Journal of Group Decision and Negotiation, 10(2):199-215, 2001.

[7] S. Kraus. Strategic Negotiation in Multi-Agent Environments. Intelligent Robots and Autonomous Agents. The MIT Press, San Francisco, CA, 2001.

[8] R. Mailler, V. Lesser, and B. Horling. Cooperative negotiation for soft real-time distributed resource allocation. In AAMAS '03: Proceedings of the second international joint conference on Autonomous agents and multiagent systems, pages 576-583, New York, NY, USA, 2003. ACM Press.

[9] S. Sariel and T. Balch. Real time auction based allocation of tasks for multi-robot exploration problem in dynamic environments. In The Twentieth National Conference on Artificial Intelligence (AAAI), Integrating Planning into Scheduling Workshop, pages 27-33, 2005.

[10] L.-K. Soh and C. Tsatsoulis. Reflective negotiating agents for real-time multisensor target tracking. In Proceedings of the International Joint Conferences on Artificial Intelligence, pages 1121-1127, 2001.

[11] F. Zeuthen. Problems of monopoly and economic warfare. Routledge and Sons, London, UK, 1930. 